\title{
Integrating multi-omics analyses of Nonomuraea dietziae to reveal the role of soybean oil in $\left[\left(4^{\prime}-\mathrm{OH}\right) \text { MeLeu }\right]^{4}-\mathrm{Cs} A$ overproduction
}

\author{
Huanhuan Liu ${ }^{1,2+}$, Di Huang ${ }^{3,4+}$, Lina Jin ${ }^{1,2+}$, Cheng Wang ${ }^{1,2}$, Shaoxiong Liang ${ }^{1,2}$ and Jianping Wen ${ }^{1,2^{*}}$
}

\begin{abstract}
Background: Nonomuraea dietziae is a promising microorganism to mediate the region-specific monooxygenation reaction of cyclosporine A (CSA). The main product [(4'-OH)MeLeu] $]^{4}-\mathrm{CSA}$ possesses high anti-HIV/HCV and hair growth-stimulating activities while avoiding the immunosuppressive effect of CsA. However, the low conversion efficiency restricts the clinical application. In this study, the production of $\left[\left(4^{\prime}-\mathrm{OH}\right) \mathrm{MeLeu}\right]^{4}$-CsA was greatly improved by $55.6 \%$ from 182.8 to $284.4 \mathrm{mg} / \mathrm{L}$ when supplementing soybean oil into the production medium, which represented the highest production of [(4'-OH)MeLeu $]^{4}-\mathrm{CsA}$ so far.

Results: To investigate the effect of soybean oil on CsA conversion, some other plant oils (corn oil and peanut oil) and the major hydrolysates of soybean oil were fed into the production medium, respectively. The results demonstrated that the plant oils, rather than the hydrolysates, could significantly improve the $\left[\left(4^{\prime}-\mathrm{OH}\right) \mathrm{MeLeu}\right]^{4}$-CSA production, suggesting that soybean oil might not play its role in the lipid metabolic pathway. To further unveil the mechanism of [(4'-OH)MeLeu $]^{4}$-CsA overproduction under the soybean oil condition, a proteomic analysis based on the two-dimensional gel electrophoresis coupled with MALDI TOF/TOF mass spectrometry was implemented. The results showed that central carbon metabolism, genetic information processing and energy metabolism were significantly up-regulated under the soybean oil condition. Moreover, the gas chromatography-mass spectrometry-based metabolomic analysis indicated that soybean oil had a great effect on amino acid metabolism and tricarboxylic acid cycle. In addition, the transcription levels of cytochrome P450 hydroxylase (CYP) genes for CsA conversion were determined by RT-qPCR and the results showed that most of the CYP genes were up-regulated under the soybean oil condition.
\end{abstract}

Conclusions: These findings indicate that soybean oil could strengthen the primary metabolism and the CYP system to enhance the mycelium growth and the monooxygenation reaction, respectively, and it will be a guidance for the further metabolic engineering of this strain.

Keywords: [(4'-OH)MeLeu] $]^{4}$-CsA, Nonomuraea dietziae, Soybean oil, Proteomics, Metabolomics, Cytochrome P450 hydroxylases

\section{Background}

Cyclosporine A (CsA), produced by soil fungus Tolypocladium niveum, is a natural cyclic undecapeptide

\footnotetext{
*Correspondence: jpwen@tju.edu.cn

${ }^{\dagger}$ Huanhuan Liu, Di Huang and Lina Jin contributed equally to this work

${ }^{1}$ Key Laboratory of System Bioengineering (Tianjin University), Ministry

of Education, Tianjin 300072, People's Republic of China

Full list of author information is available at the end of the article
}

possessing immunosuppressive activity, and is the active ingredient of Sandimmune ${ }^{\circledR}$ and Neoral ${ }^{\circledR}$ for preventing organ transplant rejection [1]. Besides, CsA is reported to substantially inhibit the virus replication of HCV (hepatitis $\mathrm{C}$ virus) [2,3] and HIV-1 (human immunodeficiency virus type 1) [4]. However, developing a CsA-based anti$\mathrm{HIV} / \mathrm{HCV}$ drug should exclude the immunosuppressive activity of CsA since it will antagonize the host immune 
system for clearing virus [5]. Fortunately, this problem has been effectively solved by modifying the side chain of the $[\mathrm{MeLeu}]^{4}$ residue [6]. Among these derivatives, $\left[\left(4^{\prime}-\mathrm{OH}\right) \mathrm{MeLeu}\right]^{4}-\mathrm{CsA}$ has a high anti-HIV/HCV activity while significantly lowering the immunosuppressive activity $[6,7]$. Meanwhile, $\left[\left(4^{\prime}-\mathrm{OH}\right) \mathrm{MeLeu}\right]^{4}-\mathrm{CsA}$ is a starting point to trigger the search for other promising analogues, e.g., $[(\mathrm{D}) \mathrm{MeSer}]^{3}-\left[\left(4^{\prime}-\mathrm{OH}\right) \mathrm{MeLeu}\right]^{4}-\mathrm{CsA}$ and $[\mathrm{Sar}-\mathrm{D}-\mathrm{OMe}]^{3}[(4-\mathrm{OH}) \mathrm{MeLeu}]^{4}-\mathrm{CsA}$, both of whose anti-HIV activities are more than sevenfold of CsA $[8,9]$. Recently, [(4'-OH)MeLeu $]^{4}-\mathrm{CsA}$ has also been reported to possess the hair growth-promoting effect [7, 10], which attracted much attention from both academy and cosmetics industry due to its potential value to treat alopecia $[11,12]$.

Nonomuraea dietziae (Sebekia benihana), a rare soil actinomycete, is one of the best microorganisms that can efficiently convert CsA to the region-specific hydroxylation product $\left[\left(4^{\prime}-\mathrm{OH}\right) \mathrm{MeLeu}\right]^{4}-\mathrm{CsA}[13]$ by cytochrome P450 hydroxylases (CYPs) [14]. To improve the bioconversion rate of CsA, various strategies have been applied so far, such as genetic manipulation [14, 15], medium optimization and traditional mutation. Lee et al. overexpressed CYP-sb21 gene in wild-type $N$. dietziae and increased the conversion rate of CsA by twofold (reaching 29\%) [16]. By the same token, a 54\% conversion rate was obtained when molybdenum salt was added into the optimized medium [17]. However, the product titers and yields of these strains are still at a low level, and impede the industrial application of $\left[\left(4^{\prime}-\mathrm{OH}\right) \mathrm{MeLeu}\right]^{4}-\mathrm{CsA}$.

Recently, we have successfully obtained a mutant strain with the high $\left[\left(4^{\prime}-\mathrm{OH}\right) \mathrm{MeLeu}\right]^{4}-\mathrm{CsA}$ production by UV$\mathrm{LiCl}$ complex mutation. Interestingly, addition of $0.1 \%$ $(\mathrm{w} / \mathrm{v})$ soybean oil could further increase the conversion rate by $55.6 \%$. Although soybean oil has been proved to accelerate the strain's growth and improve the antibiotic production [18-20], the specific effects on $\left[\left(4^{\prime}-\mathrm{OH}\right)\right.$ MeLeu $]^{4}$-CsA production may be complex and comprehensive because the conversion process from CsA to $\left[\left(4^{\prime}-\mathrm{OH}\right) \mathrm{MeLeu}\right]^{4}-\mathrm{CsA}$ is a monooxygenation reaction and seems irrelevant to soybean oil. Hence, to unveil the potential mechanism, in this study, the two-dimensional gel electrophoresis (2-DE) coupled with matrix-assisted laser-desorption/ionization time-of-flight/time-of-flight mass spectrometry (MALDI-TOF/TOF-MS) and gas chromatography-mass spectrometry (GC-MS), were employed for proteomic and metabolomic analyses, respectively. Moreover, the transcription levels of CYP genes were analyzed by quantitative real-time PCR (qRTPCR) to investigate the role of the soybean oil on the overproduction of $\left[\left(4^{\prime}-\mathrm{OH}\right) \mathrm{MeLeu}\right]^{4}-\mathrm{CsA}$.

\section{Results and discussion}

Effects of the exogenous soybean oil on the fermentation properties of $\mathrm{N}$. dietziae

As shown in Fig. 1, the major fermentation features of $N$. dietziae in soybean oil medium (assigned as the medium $\mathrm{MO}$ ) were distinctly different from the control group (medium $\mathrm{MC}$ ). The initial $12 \mathrm{~h}$ was the lag phase and the both biomass changed little, while the following log phase (12-48 h) displayed significant discrepancies (Fig. 1a). During the log phase, biomass in the medium MO increased sharply from 3.63 to $6.4 \mathrm{~g} / \mathrm{L}$ (improved by $76.3 \%$, Fig. 1a), compared with a relatively gentle change in the medium MC (improved by $43.5 \%$, Fig. 1a), indicating a more appropriate growth environment under the MO condition. Throughout the whole fermentation process, the highest biomass $(7.58 \mathrm{~g} / \mathrm{L})$ and [ $\left(4^{\prime}-\right.$ $\mathrm{OH}) \mathrm{MeLeu}]^{4}$-CsA titer $(233.4 \mu \mathrm{mol} / \mathrm{L}$ or $284.4 \mathrm{mg} / \mathrm{L})$ were achieved at $120 \mathrm{~h}$ in the medium MO, 1.16- and 1.56-folds compared to that in the medium MC, respectively (Fig. 1a, b). After $120 \mathrm{~h}$, the $\left[\left(4^{\prime}-\mathrm{OH}\right) \mathrm{MeLeu}\right]^{4}$ CsA production declined gradually due to the product degradation.

In this study, the intrinsic conversion capacity of $N$. dietziae per unit of biomass was characterized by the specific production rate $\left(\mu_{\mathrm{p}}\right)$, an important kinetic parameter that removed the impact of biomass on the $\left[\left(4^{\prime}-\mathrm{OH}\right)\right.$ MeLeu $]^{4}$-CsA production. As shown in Fig. 1c, the maximum $\mu_{\mathrm{p}}$ was achieved in the medium $\mathrm{MO}(0.67 \mu \mathrm{mol} / \mathrm{g} / \mathrm{h}$, $36 \mathrm{~h}$ ), which was 1.3 -folds and appeared $12 \mathrm{~h}$ earlier than that in the $\mathrm{MC}$ medium $(0.51 \mu \mathrm{mol} \mathrm{g} / \mathrm{h}, 48 \mathrm{~h})$. Although the $\mu_{\mathrm{p}}$ of $\mathrm{MO}$ was lower than $\mathrm{MC}$ during the late fermentation period (from $84 \mathrm{~h}$ to $120 \mathrm{~h}$, Fig. 1c), yet the most production of $\left[\left(4^{\prime}-\mathrm{OH}\right) \mathrm{MeLeu}\right]^{4}-\mathrm{CsA}$ achieved at $84 \mathrm{~h}$ in both media (Fig. 1b), indicating that the catalytic capacity of medium $\mathrm{MO}$ was superior to MC.

In addition, the concept "total mole concentration" (TMC) was put forward to describe the total mole concentration of the residual CsA and the produced $\left[\left(4^{\prime}-\mathrm{OH}\right) \mathrm{MeLeu}\right]^{4}-\mathrm{CsA}$ in the fermentation broth media based on the fact that the hydroxylation process is a monooxygenation reaction, in which the conversion coefficient of CsA to $\left[\left(4^{\prime}-\mathrm{OH}\right) \mathrm{MeLeu}\right]^{4}-\mathrm{CsA}$ is $1 \mathrm{~mol}: 1 \mathrm{~mol}$. In Fig. 1d, the TMC reached the minimum value at $48 \mathrm{~h}$, but significantly increased during the late fermentation period in both medium. This phenomenon indicated that part of CsA was probably converted into other intermediates, such as the CsA-CYPs complex, as mentioned previously $[16,21]$. Accordingly, the lower value of TMC in the MO medium represented a stronger intermediate processing capacity (or catalytic ability) than that in the control. 

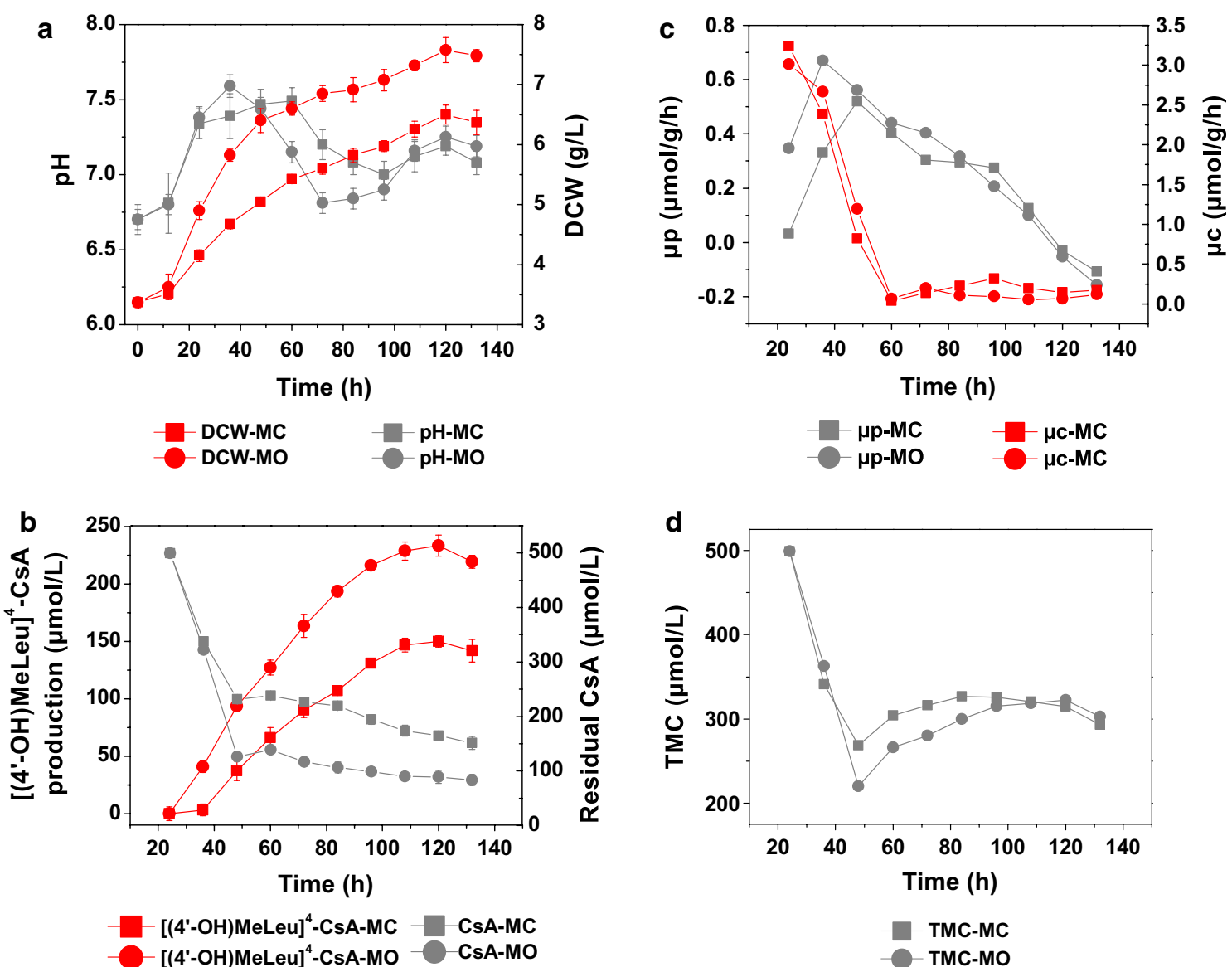

Fig. 1 Time course profiles of key fermentation parameters in $500 \mathrm{~mL}$ shaking flask. a Dynamic fermentation profiles of dry cell weight and pH; b Concentration of $\left[\left(4^{\prime}-\mathrm{OH}\right) \mathrm{MeLeu}\right]^{4}-\mathrm{CSA}$ and CSA in medium MO and MC; $\mathbf{c}$ Changes of the specific rates in medium MO and MC; $\mathbf{d}$ The total mole concentration of CSA and $\left[\left(4^{\prime}-\mathrm{OH}\right) \mathrm{MeLeu}\right]^{4}-\mathrm{CsA}$. Each value represents the mean of five independent experiments and the error bars represent standard deviations of five values. $M O$ soybean oil medium, $M C$ soybean oil-free medium; $\mu_{p}$, specific production rate of $\left[\left(4^{\prime}-\mathrm{OH}\right) \mathrm{MeLeu}\right]^{4}$-CsA, $\mu_{c}$ specific consumption rate of CSA, TMC total mole concentration of CSA and [(4'-OH)MeLeu $]^{4}$-CSA

Effects of the major hydrolysates of soybean oil and some other plant oils (corn oil and peanut oil) on [(4'-OH) MeLeu] $]^{4}-$ CsA production

Since soybean oil is a natural lipid mixture and can be hydrolyzed into fatty acids and glycerol, both of which will subsequently participate in the metabolic system $[19,22]$. Considering the potential association between the lipid metabolism and the soybean oil, series of feeding experiments were designed and carried out, including the main hydrolysates of soybean oil, such as oleic acid, linoleic acid and glycerol, as well as the soybean oil-like compounds, such as corn oil $(0.1 \%$, w/v), peanut oil $(0.1 \%, w / v)$ and Tween 80 (polyoxyethylene sorbitan monooleate, $0.05 \%, \mathrm{w} / \mathrm{v})$. All the compounds were fed into the medium $\mathrm{MC}$ at the beginning of fermentation.

As shown in Fig. 2, oleic acid, linoleic acid and Tween 80 all failed to enhance the conversion of CsA to [(4'-OH)
MeLeu $]^{4}$-CsA. Glycerol was an exception since the [(4'$\mathrm{OH}) \mathrm{MeLeu}]^{4}$-CsA production had a $7 \%$ increase. Considering that glycerol was probably used as a complementary carbon source, and that increasing the concentration of glycerol could not further enhance the CsA conversion (data not shown), a 7\% increase, nevertheless, was still much smaller compared with the impact of soybean oil (55.6\%). Thus, it could be concluded that the main hydrolysates of soybean oil contributed little or even negatively to the improvement of CsA conversion.

Addition of corn oil and peanut oil, by contrast, led to the 20.7 and $25.8 \%$ increases in $\left[\left(4^{\prime}-\mathrm{OH}\right) \mathrm{MeLeu}\right]^{4}-\mathrm{CsA}$ production, respectively, suggesting that plant oils (soybean oil, corn oil and peanut oil) could efficiently improve the conversion of CsA. Therefore, although $N$. dietziae cannot directly utilize the hydrolysates of plant oil to strengthen mycelium growth and $\left[\left(4^{\prime}-\mathrm{OH}\right) \mathrm{MeLeu}\right]^{4}-\mathrm{CsA}$ 


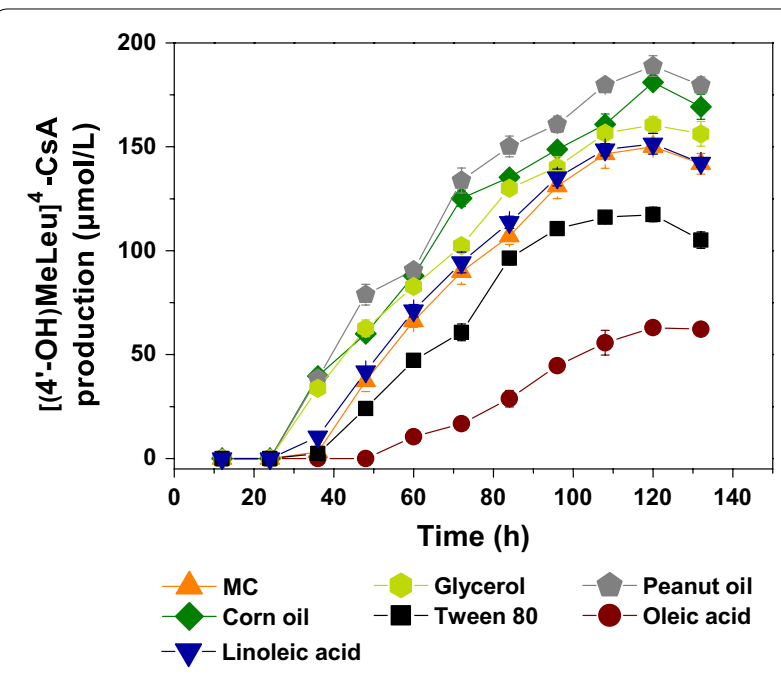

Fig. 2 Effects of major hydrolysates of soybean oil and some other plant oils (corn oil and peanut oil) on [(4'-OH)MeLeu $]^{4}-\mathrm{CsA}$ production. Each value represents the mean of five independent experiments and the error bars represent standard deviations of five values

synthesis, the overproduction of $\left[\left(4^{\prime}-\mathrm{OH}\right) \mathrm{MeLeu}\right]^{4}-\mathrm{CsA}$ indicated that plant oils worked in another way rather than being the substrates.

Taken the above results, soybean oil played a significant role in improving biomass and specific production rate. However, soybean oil might not play its role in the lipid metabolic pathway and more intercellular metabolic details still remained to be investigated. To this end, a combined proteomic and metabolic analysis was implemented to further reveal the role of soybean oil in $\left[\left(4^{\prime}-\right.\right.$ $\mathrm{OH}) \mathrm{MeLeu}]^{4}$-CsA overproduction.

\section{Comparative proteomic and metabolomic analyses in response to the soybean oil addition Comparative proteomic profile analysis}

Protein extracts from both conditions were sampled at $48 \mathrm{~h}$ and $96 \mathrm{~h}$ and then subjected to 2-DE. The results of gel electrophoresis were presented in Additional file 1: Figure S4 and 2-DE could effectively separate most of the proteins, indicating the feasibility of our experimental methods. In this study, a total of 95 protein spots in the 2-DE gels with significantly differential expressions (fold change $>1.5$ ) were identified (Table 1 ). They were classified into eight functional groups by their cellular roles, mainly including central carbon metabolism, energy metabolism, genetic information processing, amino acid metabolism, regulatory proteins, nucleotide metabolism, proteins of unknown function and hypothetical proteins. Figure 3 presented the protein distribution at the sampling time with different abundances, and most of the proteins in each group were present at higher levels under the MO condition, suggesting a comprehensive strengthening effect of soybean oil on the whole cell metabolism.

\section{Comparative metabolic profile analysis}

Intercellular metabolites of $N$. dietziae (48, 72, 96 and $120 \mathrm{~h})$ in the medium $\mathrm{MO}$ and MC with different [(4'$\mathrm{OH}) \mathrm{MeLeu}]^{4}$-CsA producing capabilities were analyzed by GC-MS. As a result, a total of 50 intracellular metabolites were identified, including amino acids, organic acids, sugars and fatty acids. Abundance changes of metabolites are depicted by the heat map in Fig. 4.

The metabolomic profiling showed obvious differences in tricarboxylic acid (TCA) cycle and amino acid metabolism. More specifically, succinic acid increased to 2.8- and 5.5-fold of that in the medium MC at 48 and $96 \mathrm{~h}$, respectively. Similar change patterns also exhibited in fumarate (1.4- and 1.2-fold vs. the control) and $\alpha$-ketoglutaric acid (1.2- and 2.2-fold vs. the control). Although the abundances of these metabolites decreased gradually along the fermentation process, they were still much higher than the control, indicating the enhancement of TCA cycle in medium MO. On the contrary, the abundance of amino acids such as threonine, glutamate, lysine, valine and leucine were relatively lower in medium MO. Particularly, both glutamate and lysine decreased by $78 \%$ at $72 \mathrm{~h}$. A reasonable explanation was that large amount of amino acids was effectively consumed to synthesize cellular building blocks for cell growth.

Based on the proteomic and metabolomic results, we therefore summarized the metabolic profile in Fig. 5, which visually described the intracellular metabolic pathway in response to the exogenous soybean oil. In this model, most proteins with high levels (in red font) were mainly involved in the central carbon metabolism, amino acid metabolism, genetic information processing and oxidation-reduction process. As regarding to the hydroxylation of CsA to $\left[\left(4^{\prime}-\mathrm{OH}\right) \mathrm{MeLeu}\right]^{4}-\mathrm{CsA}$, it was directly mediated by CYPs and P450 oxidoreductase (POR) along with the formation of water and $\operatorname{NAD}(\mathrm{P})^{+}$, which was seemingly uncorrelated to other intracellular metabolic activities. Subsequently, pathway analysis was implemented to dissect the effects of soybean oil on the metabolism and regulation of $N$. dietziae in detail.

\section{Central carbon metabolism and amino acid metabolism}

Central carbon metabolism represents the backbone of the cellular metabolism and provides the precursors required for the cell growth and the synthesis of target products. As shown in Fig. 5, it was worth noting that some key enzymes involved in glycolytic pathway, such as 6-phosphofructokinase (Spot 6, PFK) and pyruvate kinase (Spot 8, PYK) were both present at higher levels. 
Table 1 Differentially expressed proteins identified by MALDI-TOF/TOF-MS under the soybean oil and the control conditions

\begin{tabular}{|c|c|c|c|c|c|c|c|c|c|}
\hline Spot no. ${ }^{a}$ & Protein name & Species & $\begin{array}{l}\text { NCBI access- } \\
\text { ion no. }\end{array}$ & $\begin{array}{l}\text { Protein } \\
\text { MW }^{c}\end{array}$ & Protein $\mathrm{PI}$ & $\begin{array}{l}\text { Protein } \\
\text { score }^{d}\end{array}$ & $\begin{array}{l}\text { Protein } \\
\text { score C.I. \% }\end{array}$ & $\begin{array}{l}\text { MO/MC } \\
(48 h)^{\mathrm{e}}\end{array}$ & $\begin{array}{l}\text { MO/MC } \\
(96 \mathrm{~h})\end{array}$ \\
\hline 1 & $\begin{array}{l}\text { Glucose-6-phosphate } \\
\text { dehydrogenase }\end{array}$ & Nonomuraea sp. SBT364 & gi|898235202 & 56.92 & 5.96 & 138 & 100 & 1.67 & 0.56 \\
\hline 2 & $\begin{array}{l}\text { Phosphogluconate dehy- } \\
\text { dratase }\end{array}$ & Nonomuraea sp. SBT364 & gi|898253514 & 68.06 & 5.8 & 67 & 99.5 & Loss & 1.64 \\
\hline 3 & Enolase & $\begin{array}{l}\text { Streptosporangium } \\
\text { amethystogenes }\end{array}$ & gi|664384366 & 45.33 & 4.53 & 72 & 99.76 & 1.78 & 1.89 \\
\hline 4 & $\begin{array}{l}\text { 3-phosphoglycerate dehy- } \\
\text { drogenase }\end{array}$ & Nonomuraea candida & gi|759933157 & 55.53 & 4.99 & 135 & 100 & 1.96 & 1.87 \\
\hline 5 & $\begin{array}{l}\text { Dihydrolipoamide dehydro- } \\
\text { genase }\end{array}$ & Nonomuraea candida & gi|759953882 & 47.83 & 5.47 & 62 & 98.23 & 1.93 & 1.21 \\
\hline 6 & 6-phosphofructokinase & Nonomuraea coxensis & gi|648522261 & 36.66 & 5.53 & 125 & 100 & 2.17 & 1.74 \\
\hline 7 & Pyruvate dehydrogenase & Nonomuraea candida & gi|759929120 & 102.11 & 5.83 & 146 & 100 & 2.35 & 1.85 \\
\hline 8 & Pyruvate kinase & Nonomuraea candida & gi|759954395 & 51.48 & 5.94 & 118 & 100 & 2.05 & 1.78 \\
\hline 9 & Malate dehydrogenase & Nonomuraea sp. SBT364 & gi|898218461 & 34.1 & 4.85 & 65 & 99.23 & 1.75 & 1.64 \\
\hline 10 & $\begin{array}{l}\text { 2-oxoglutarate ferredoxin } \\
\text { oxidoreductase subunit } \\
\text { alpha }\end{array}$ & Nonomuraea sp. SBT364 & gi|898257150 & 65.95 & 5.34 & 76 & 99.94 & 1.68 & 0.77 \\
\hline 11 & Citrate synthase & Nonomuraea candida & gi|759936001 & 40.74 & 5.45 & 67 & 99.25 & 3.32 & 1.71 \\
\hline 12 & $\begin{array}{l}\text { Succinyl-CoA synthetase } \\
\text { subunit alpha }\end{array}$ & Nonomuraea sp. SBT364 & gi|898242487 & 30.38 & 6.1 & 131 & 100 & 2.45 & 1.78 \\
\hline 13 & $\begin{array}{l}\text { Alpha-ketoglutarate decar- } \\
\text { boxylase }\end{array}$ & Nonomuraea candida & gi|759939264 & 134.27 & 5.91 & 103 & 100 & 2.19 & 1.62 \\
\hline 14 & $\begin{array}{l}\text { Cyclopropane-fatty-acyl- } \\
\text { phospholipid synthase }\end{array}$ & Nonomuraea candida & gi|759945365 & 46.94 & 6.07 & 67 & 99.35 & 1.62 & 0.72 \\
\hline 15 & $\begin{array}{l}\text { Cytochrome P450 hydroxy- } \\
\text { lase sb15 }\end{array}$ & Nonomuraea dietziae & gi|445067401 & 51.04 & 8.82 & 34 & 99.11 & 1.73 & 1.67 \\
\hline 16 & 2-isopropylmalate synthase & Nonomuraea sp. SBT364 & gi|898222194 & 63.16 & 5.04 & 187 & 100 & 1.25 & 1.84 \\
\hline 17 & $\begin{array}{l}\text { Cytochrome P450 hydroxy- } \\
\text { lase sb8 }\end{array}$ & Nonomuraea dietziae & gi|445067389 & 44.43 & 5.23 & 25 & 97.84 & 1.85 & 0.82 \\
\hline 18 & $\begin{array}{l}\text { Cytochrome P450 hydroxy- } \\
\text { lase sb17 }\end{array}$ & Nonomuraea dietziae & gi|445067405 & 43.62 & 5.19 & 25 & 97.37 & 2.06 & 0.71 \\
\hline 19 & $\begin{array}{l}\text { Cytochrome P450 hydroxy- } \\
\text { lase sb2 }\end{array}$ & Nonomuraea dietziae & gi|445067377 & 25.04 & 5.5 & 33 & 98.85 & 1.76 & 1.22 \\
\hline 20 & $\begin{array}{l}\text { Cytochrome } \mathrm{P} 450 \text { hydroxy- } \\
\text { lase sb20 }\end{array}$ & Nonomuraea dietziae & gi|445067407 & 42.77 & 5.05 & 34 & 99.15 & 1.95 & 1.65 \\
\hline 21 & NADH dehydrogenase & Nonomuraea candida & gi|759944851 & 49.06 & 5.22 & 112 & 100 & 3.39 & 0.97 \\
\hline 22 & Acyl-CoA thioesterase & $\begin{array}{l}\text { Alicyclobacillus acidoter- } \\
\text { restris }\end{array}$ & gi|916582360 & 17.26 & 5.58 & 89 & 96.85 & 0.61 & 1.68 \\
\hline 23 & NADP oxidoreductase & Nonomuraea coxensis & gi|916408869 & 47.19 & 6.08 & 71 & 99.79 & 1.87 & 2.15 \\
\hline 24 & $\begin{array}{l}\text { Flavoprotein disulfide } \\
\text { reductase }\end{array}$ & $\begin{array}{l}\text { Streptosporangium } \\
\text { amethystogenes }\end{array}$ & gi|664385831 & 48.56 & 5.37 & 69 & 99.55 & 2.08 & 1.75 \\
\hline 25 & $\begin{array}{l}\text { NADH dehydrogenase } \\
\text { subunit F }\end{array}$ & $\begin{array}{l}\text { Streptosporangium } \\
\text { roseum }\end{array}$ & gi|502651094 & 46.67 & 5.5 & 115 & 100 & 0.68 & 1.94 \\
\hline 26 & FAD-linked oxidase & Nonomuraea sp. SBT364 & gi|898280523 & 55.07 & 5.87 & 92 & 100 & 1.72 & 1.98 \\
\hline 27 & $\begin{array}{l}\text { Flavoprotein oxidoreduc- } \\
\text { tase }\end{array}$ & $\begin{array}{l}\text { Streptosporangium } \\
\text { roseum }\end{array}$ & gi|502655712 & 48.81 & 5.58 & 60 & 99.55 & 1.79 & 1.73 \\
\hline 28 & $\begin{array}{l}\text { Transcription termination } \\
\text { factor NusA }\end{array}$ & Nonomuraea coxensis & gi|522034561 & 36.32 & 5.43 & 87 & 99.99 & 2.27 & 1.17 \\
\hline 29 & MFS transporter & $\begin{array}{l}\text { Streptosporangium } \\
\text { amethystogenes }\end{array}$ & gi|664381342 & 79.76 & 6.34 & 57 & 97.78 & 1.64 & 1.32 \\
\hline 30 & Thiosulfate sulfurtransferase & Nonomuraea coxensis & gi|522033492 & 31.21 & 4.81 & 62 & 98.19 & 1.74 & 1.94 \\
\hline 31 & $\begin{array}{l}\text { Sulfonate ABC transporter } \\
\text { ATP-binding protein }\end{array}$ & Nonomuraea candida & gi|759950693 & 25.17 & 7 & 55 & 97.5 & 1.80 & 1.68 \\
\hline
\end{tabular}


Table 1 continued

\begin{tabular}{|c|c|c|c|c|c|c|c|c|c|}
\hline Spot no. ${ }^{a}$ & Protein name & Species & $\begin{array}{l}\text { NCBI access- } \\
\text { ion no. }\end{array}$ & $\begin{array}{l}\text { Protein } \\
\text { MW }^{c}\end{array}$ & Protein PI & $\begin{array}{l}\text { Protein } \\
\text { score }^{d}\end{array}$ & $\begin{array}{l}\text { Protein } \\
\text { score C.I. \% }\end{array}$ & $\begin{array}{l}\mathrm{MO} / \mathrm{MC} \\
(48 \mathrm{~h})^{\mathrm{e}}\end{array}$ & $\begin{array}{l}\text { MO/MC } \\
(96 \mathrm{~h})\end{array}$ \\
\hline 32 & ATP-binding protein & Nonomuraea coxensis & gi|648523005 & 48.21 & 5.74 & 150 & 100 & 1.67 & 0.88 \\
\hline 33 & $\begin{array}{l}\text { S-adenosylmethionine } \\
\text { synthetase }\end{array}$ & $\begin{array}{l}\text { Streptosporangium } \\
\text { amethystogenes }\end{array}$ & gi|664383325 & 42.57 & 4.98 & 120 & 100 & 2.04 & 1.17 \\
\hline 34 & $\begin{array}{l}\text { S-adenosyl-L-homocysteine } \\
\text { hydrolase }\end{array}$ & Nonomuraea coxensis & gi|522030148 & 52.1 & 5.33 & 91 & 100 & 1.66 & 4.28 \\
\hline 35 & Glutamate synthase & Nonomuraea coxensis & gi|703367593 & 160.46 & 5.48 & 79 & 99.96 & 2.47 & 0.62 \\
\hline 36 & $\begin{array}{l}\text { Phenylalanine-tRNA ligase } \\
\text { subunit alpha }\end{array}$ & Nonomuraea coxensis & gi|522035904 & 38.25 & 5.4 & 93 & 100 & 1.74 & 1.32 \\
\hline 37 & $\begin{array}{l}\text { Aspartyl/glutamyl-tRNA } \\
\text { amidotransferase subunit } \\
\text { B }\end{array}$ & $\begin{array}{l}\text { Streptosporangium } \\
\text { roseum }\end{array}$ & gi|502658302 & 54.44 & 5.13 & 117 & 100 & 1.88 & 2.38 \\
\hline 38 & Proline-tRNA ligase & Nonomuraea candida & gi|759956543 & 63.49 & 5.24 & 107 & 100 & 11.29 & 2.46 \\
\hline 39 & $\begin{array}{l}\text { Tryptophanyl-tRNA syn- } \\
\text { thetase }\end{array}$ & Nonomuraea sp. SBT364 & gi|898218468 & 37.51 & 6.21 & 100 & 100 & 0.45 & 1.46 \\
\hline 40 & Cysteine synthase & Nonomuraea coxensis & gi|522035423 & 34.18 & 5.47 & 70 & 99.75 & 2.45 & 1.45 \\
\hline 41 & ATPase AAA & $\begin{array}{l}\text { Lachnoanaerobaculum } \\
\text { sp. ICM7 }\end{array}$ & gi|497349325 & 83.91 & 5.1 & 186 & 100 & 1.82 & 1.63 \\
\hline 42 & Urocanate hydratase & Nonomuraea coxensis & gi|703370540 & 59.91 & 5.54 & 75 & 99.9 & 1.71 & 2.08 \\
\hline 43 & Histidine ammonia-lyase & Nonomuraea sp. SBT364 & gi|898282330 & 53.36 & 5.34 & 67 & 99.4 & 1.74 & 0.97 \\
\hline 44 & $\begin{array}{l}\text { Inosine-5-monophosphate } \\
\text { dehydrogenase }\end{array}$ & Nonomuraea sp. SBT364 & gi|759938464 & 39.48 & 5.74 & 150 & 100 & 2.02 & 1.76 \\
\hline 45 & $\begin{array}{l}\text { Polynucleotide phosphory- } \\
\text { lase }\end{array}$ & Nonomuraea coxensis & gi|522034552 & 84.03 & 5.11 & 158 & 100 & 0.76 & 1.62 \\
\hline 46 & Elongation factor Ts & $\begin{array}{l}\text { Streptosporangium } \\
\text { roseum }\end{array}$ & gi|502652347 & 30.18 & 5.27 & 121 & 100 & 9.17 & 0.89 \\
\hline 47 & $\begin{array}{l}\text { DNA-directed RNA poly- } \\
\text { merase subunit alpha }\end{array}$ & $\begin{array}{l}\text { Alkalibacillus haloalka- } \\
\text { liphilus }\end{array}$ & gi|515752615 & 35.17 & 4.8 & 282 & 96.64 & 1.61 & 0.69 \\
\hline 48 & $\begin{array}{l}\text { DNA polymerase III subunit } \\
\text { beta }\end{array}$ & Nonomuraea sp. SBT364 & gi|898216335 & 40.14 & 4.79 & 215 & 100 & 1.67 & 0.73 \\
\hline 49 & 30 S ribosomal protein S2 & Nonomuraea sp. SBT364 & gi|898235817 & 34.47 & 5.02 & 161 & 100 & 1.78 & 1.62 \\
\hline 50 & $\begin{array}{l}\text { Transcription termination } \\
\text { factor Rho }\end{array}$ & Nonomuraea sp. SBT364 & gi|898215154 & 73.6 & 8.73 & 140 & 100 & 1.62 & 0.49 \\
\hline 51 & $\begin{array}{l}\text { Dihydrolipoyl dehydroge- } \\
\text { nase }\end{array}$ & Nonomuraea sp. SBT364 & gi|898266057 & 49.7 & 5.51 & 126 & 100 & 1.74 & 1.85 \\
\hline 52 & $\begin{array}{l}\text { Peptide chain release } \\
\text { factor } 2\end{array}$ & Nonomuraea candida & gi|759935368 & 41.51 & 4.69 & 109 & 100 & 0.65 & 1.94 \\
\hline 53 & Molecular chaperone GroEL & Nonomuraea candida & gi|759948935 & 57.13 & 4.84 & 166 & 100 & 0.42 & 1.32 \\
\hline 54 & Histidine kinase & Nonomuraea coxensis & gi|703366436 & 148.43 & 4.97 & 267 & 100 & 1.74 & 1.36 \\
\hline 55 & Elongation factor $P$ & Nonomuraea coxensis & gi|522034280 & 19.93 & 5.00 & 130 & 100 & 0.45 & 0.90 \\
\hline 56 & $\begin{array}{l}\text { ATP-dependent Clp pro- } \\
\text { tease proteolytic subunit }\end{array}$ & Nonomuraea candida & gi|759955675 & 23.2 & 5.03 & 108 & 100 & 0.70 & 1.67 \\
\hline 57 & $\begin{array}{l}\text { Two-component system } \\
\text { sensor histidine kinase }\end{array}$ & Nonomuraea candida & gi|759950142 & 37.01 & 9.94 & 59 & 96.39 & 1.68 & 1.51 \\
\hline 58 & Proteasome subunit alpha & Nonomuraea coxensis & gi|916409057 & 30.52 & 4.99 & 91 & 100 & 1.71 & 0.76 \\
\hline 59 & ATPase AAA & Nonomuraea sp. SBT364 & gi|898282349 & 65.47 & 5.04 & 186 & 100 & 1.07 & 1.75 \\
\hline 60 & $\begin{array}{l}\text { Carbamoyl phosphate } \\
\text { synthase large subunit }\end{array}$ & Nonomuraea sp. SBT364 & gi|898274197 & 117.27 & 4.79 & 139 & 100 & 1.64 & 0.96 \\
\hline 61 & $\begin{array}{l}\text { LysR family transcriptional } \\
\text { regulator }\end{array}$ & $\begin{array}{l}\text { Streptosporangium } \\
\text { roseum }\end{array}$ & gi|759969058 & 33.7 & 6.28 & 62 & 98.03 & 0.93 & 1.05 \\
\hline 62 & $\begin{array}{l}\text { DNA-binding response } \\
\text { regulator }\end{array}$ & Nonomuraea coxensis & gi|522033451 & 25.6 & 5.17 & 69 & 99.64 & 1.69 & 0.90 \\
\hline 63 & Hypothetical protein & Kiloniella laminariae & gi|759750848 & 27.62 & 10.15 & 115 & 100 & 2.69 & 0.81 \\
\hline 64 & $\begin{array}{l}\text { Gamma-aminobutyralde- } \\
\text { hyde dehydrogenase }\end{array}$ & Nonomuraea candida & gi|759930674 & 49.16 & 5.26 & 107 & 100 & 1.68 & 1.74 \\
\hline
\end{tabular}


Table 1 continued

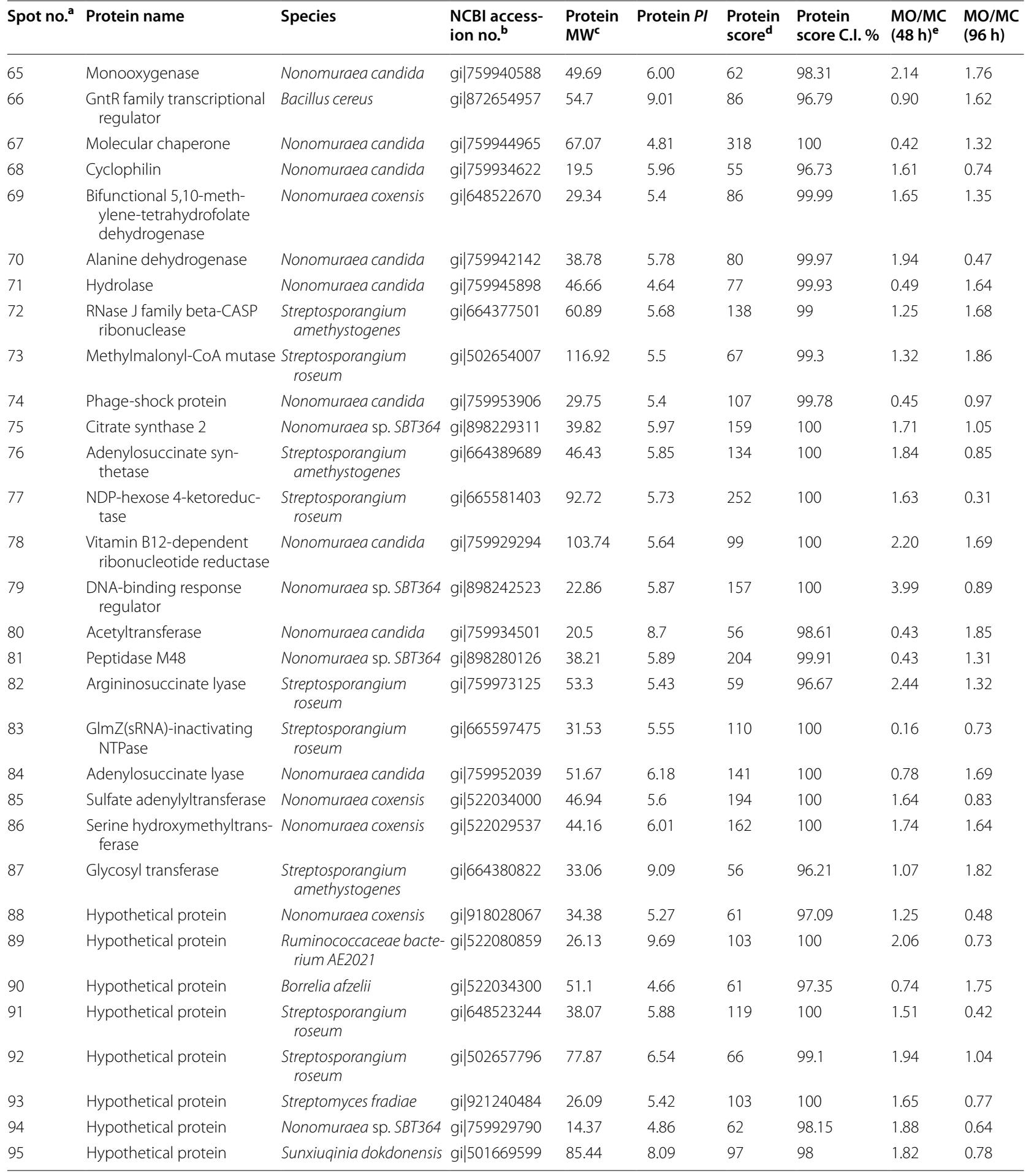

a Spot number of the differentially protein in Fig. 2

b Accession numbers in the NCBInr database

c Theoretical molecular weight $(\mathrm{kDa})$ and isoelectric point ( $\mathrm{pl}$ )

d Mascot score from the NCBInr

e Mean fold change is the ratio of protein abundance between medium MO and MC at 48 and $96 \mathrm{~h}$, respectively 


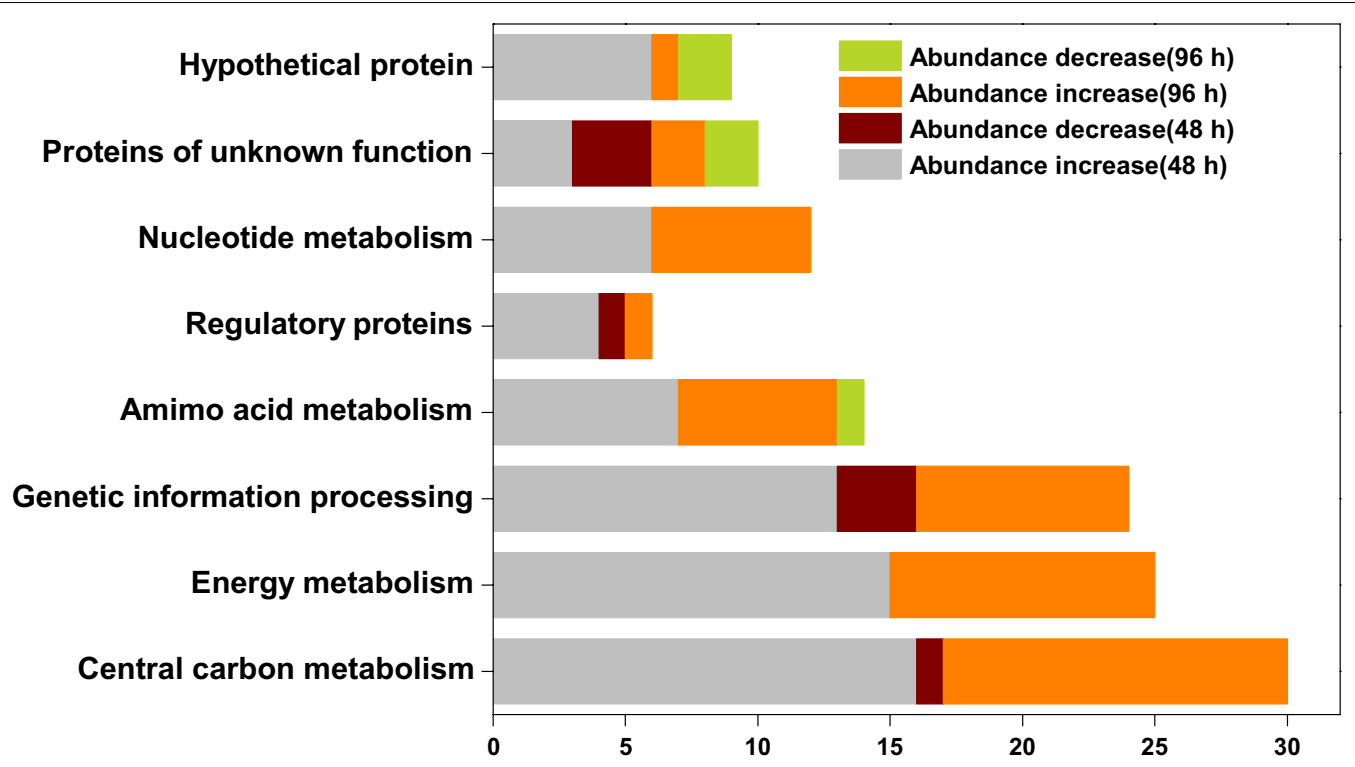

Fig. 3 Protein distribution with significant changes in each functional category at 48 and $96 \mathrm{~h}$ respectively. 2-DE profiles of $\mathrm{N}$. dietziae were presented in Additional file 1: Figure S4 and the significantly differential proteins and their characteristics were listed in Table 1

Different from many other actinomycetes, PFK of Nonomuraea is not allosterically regulated by ATP, AMP, ADP, or phosphoenolpyruvate and pyruvate, but controlled by the availability of pyrophosphate (PPi) produced in nucleic acid and protein biosynthesis and in the cycling between glycogen and glucose-1-phosphate [23]. Additionally, the activity of PPi-dependent PFK is reversible, suggesting that $N$. dietziae had a flexible glycolytic pathway as the regulatory node. Apart from PFK, dihydrolipoamide dehydrogenase (Spot 5, DLD), pyruvate dehydrogenase (Spot 7, PDH) and enolase (Spot 3, $\mathrm{ENO}$ ) also showed higher levels under the MO condition, suggesting a strengthened Embden-Meyerhof-Parnas (EMP) pathway.

In regard to TCA cycle, citrate synthase (Spot 11, CS), which converts acetyl-CoA and oxaloacetate to citrate in the initial step and controls flux into the TCA cycle [24], showed a higher level (3.23-folds) under the MO condition while the increase in the level of malate dehydrogenase (Spot 9, Mdh) could supply more oxaloacetate that reacts with acetyl-CoA [25]. In addition, the higher levels of alpha-ketoglutarate decarboxylase (Spot 13, SucA) and dihydrolipoyl dehydrogenase (Spot 51, DLDH) further improved the metabolic rate of TCA cycle [26]. As a consequence, TCA-related metabolites, such as fumarate, $\alpha$-ketoglutaric acid and succinic acid, were all present at higher levels (Fig. 4), indicating that the TCA cycle was active under the MO condition from both proteomic and metabolomic insights.
Additionally, glucose-6-phosphate dehydrogenase (Spot 1, G6PD), the first key enzyme of pentose phosphate (PP) pathway, had a higher level (1.67-fold) at $48 \mathrm{~h}$ under the MO oil condition, but at $96 \mathrm{~h}$ the abundance of G6PD decreased to $56 \%$ of the control. G6PD-catalyzed reaction dictates and limits the fluxes between EmbdenMeyerhof-Parnas (EMP) and PP pathways [27], and the $\mathrm{PP}$ pathway oxidizes glucose to generate NADPH for reductive biosynthesis reactions and ribose-5-phosphate for the synthesis of the nucleotides [28]. The decreased level of G6PD under the MO condition restricted the specific cell growth (Fig. 1, $96 \mathrm{~h}$ ) and the specific production rate of $\left[\left(4^{\prime}-\mathrm{OH}\right) \mathrm{MeLeu}\right]^{4}-\mathrm{CsA}$ (Fig. 1, $96 \mathrm{~h}$ ) since the cofactor NADPH also participated in the CsA hydroxylation process in addition to biomass accumulation.

Another significantly changed protein, phosphogluconate dehydratase (Spot 2, Edd), was only detected at $96 \mathrm{~h}$ and had a $64 \%$ increase after the soybean oil addition. Gunnarsson et al. had identified the ED pathway in Nonomuraea sp. by the ${ }^{13} \mathrm{C}$ labelling-based method [29]. ED pathway catabolizes glucose to pyruvate by using the 6-phosphogluconate dehydratase (Edd) and 2-keto-3-deoxyphosphogluconate aldolase (KDPG aldolase), which connects the EMP, PP pathways and TCA cycle, and tunes the carbon flux distribution, the production of ATP, reducing equivalent for cell growth. As the first critical enzyme of ED pathway, the higher level of Edd under the MO condition probably indicated a more robust sugar metabolism at the end of fermentation than the control. 


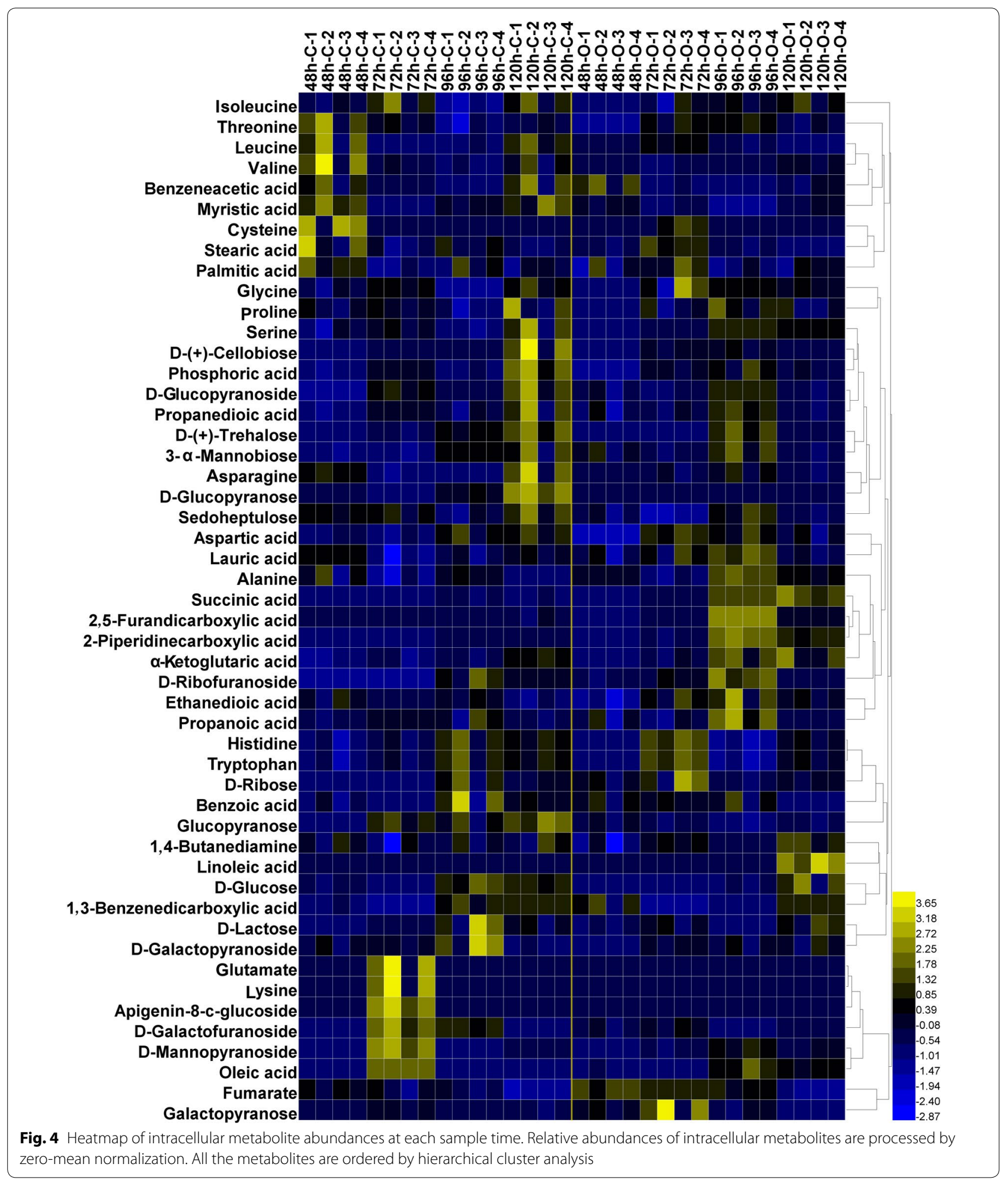

Amino acid metabolism is essential for cell growth by supplying the building blocks and metabolic intermediates along with EMP, TCA and PPP. In this study, three proteins involved in glutamate synthesis, i.e. Spot 42 (urocanate hydratase, HutU), Spot 43 (histidine ammonia-lyase, HAL) and Spot 35 (glutamate synthase, GS), as 


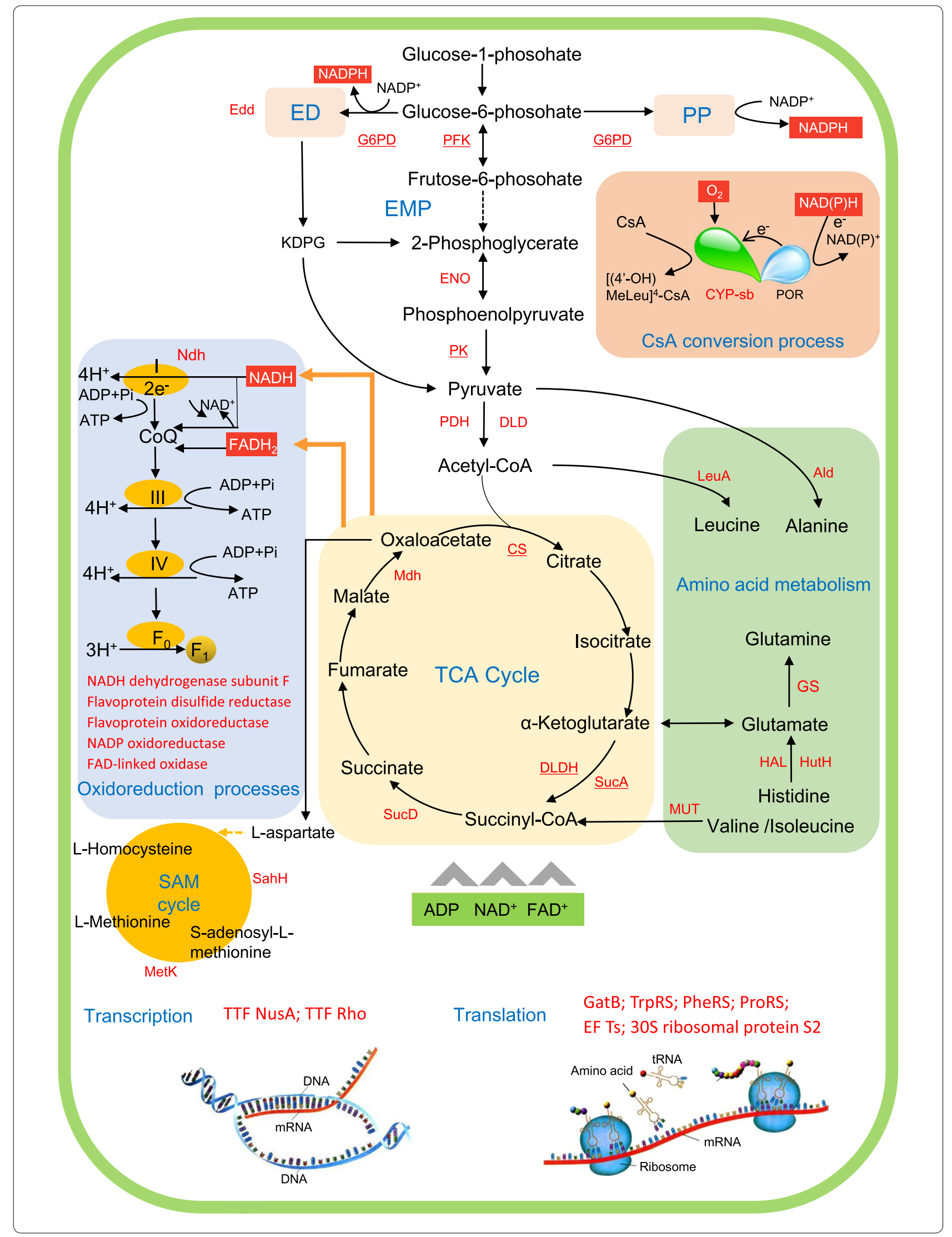


(See figure on previous page.)

Fig. 5 Scheme of metabolic pathways in $\left[\left(4^{\prime}-\mathrm{OH}\right) \mathrm{MeLeu}\right]^{4}-\mathrm{Cs} A$ production under MO condition. Enzymes in red font are activated under MO condition and the rate-limiting enzymes are underlined besides. Regions of different colors represent different metabolic modules. G6PD glucose-6-phosphate dehydrogenase, Edd phosphogluconate dehydratase, KDPG 2-keto-3-deoxy-6-phosphogluconate, PFK 6-phosphofructokinase, ENO enolase, $P K$ pyruvate kinase, DLD dihydrolipoamide dehydrogenase, $P D H$ pyruvate dehydrogenase, CS citrate synthase, $D L D H$ dihydrolipoyl dehydrogenase, SucA alpha-ketoglutarate decarboxylase, SucD succinyl-CoA synthetase alpha subunit, Ndh NADH dehydrogenase, Ald alanine dehydrogenase, LeuA 2-isopropylmalate synthase, GS glutamate synthase, HutU urocanate hydratase, HAL histidine ammonia-lyase, MUT methylmalonyl-CoA mutase, MetK S-adenosylmethionine synthetase, SahH S-adenosyl-L-homocysteine hydrolase, CYP cytochrome P450 hydroxylase, POR P450 oxidoreductase, PheRS phenylalanine-tRNA ligase, GatB aspartyl/glutamyl-tRNA amidotransferase subunit B, ProRS proline-tRNA ligase, TrpRS tryptophanyl-tRNA synthetase, EF-Ts elongation factor Ts

well as some other amino acid metabolism-related proteins (Spot 16, 2-isopropylmalate synthase, LeuA, Spot 40 , cysteine synthase, CysO) had significantly higher levels in the medium MO. Although abundances of glutamate, leucine and alanine were much lower in the medium MO than that in the medium MC (Fig. 4), it could probably be resulted from the effective utilization for protein synthesis and cell growth. In addition, two proteins in S-adenosyl-L-methionine (SAM) cycle, i.e. Spot 33 (SAM synthetase, MetK) and Spot 34 (S-adenosyl-L-homocysteine hydrolase, $\mathrm{SahH}$ ) were detected at higher abundance in the medium MO, which were involved in the synthesis of $\mathrm{Fe}-\mathrm{S}$ protein clusters and methionine [30].

Therefore, it can be concluded that addition of soybean oil could significantly upregulate the intracellular central carbon metabolism and amino acid metabolism, which supplies a more suitable intracellular environment for maintaining the cell robustness and promoting the [( $4^{\prime}-$ $\mathrm{OH}) \mathrm{MeLeu}]^{4}$-CsA overproduction.

\section{Genetic information processing and nucleotide metabolism}

Bacterial growth is directly correlated to the synthesis of protein and DNA. In this study, the higher abundances of phenylalanine-tRNA ligase (PheRS, Spot 36), aspartyl/ glutamyl-tRNA amidotransferase subunit B (GatB, Spot 37), proline-tRNA ligase (ProRS, Spot 38) and tryptophanyl-tRNA synthetase (TrpRS, Spot 39) were observed under the $\mathrm{MO}$ condition, indicating that aminoacyltRNA biosynthesis was activated. Additionally, elongation factor Ts (EF-Ts, Spot 46) and 30S ribosomal protein S2 (Spot 49) were also present at higher levels compared with the control. EF-Ts mediates the regeneration of EF-Tu-GDP complex, which catalyzes the addition of aminoacyl-tRNA into ribosome [31, 32]. Meanwhile, DNA-directed RNA polymerase subunit alpha (Spot 47), DNA polymerase III subunit beta (Spot 48), transcription termination factor NusA (Spot 28) and transcription termination factor Rho (Spot 50), were present at higher levels. They are involved in the transcription and replication processes and inosine-5-monophosphate dehydrogenase (Spot 44) provides precursors (guanine) for RNA and DNA synthesis [33]. Higher levels of the above proteins could efficiently enhance the cell growth, leading to a higher specific growth rate under the MO condition as shown in Fig. 1.

\section{Cytochrome P450 (CYP) and energy metabolism}

CYPs belong to a family of terminal monooxygenases that transfer one oxygen atom to $\mathrm{X}-\mathrm{H}$ bonds $(\mathrm{X}:-\mathrm{C},-\mathrm{N}$, S) of a substrate with the concomitant reduction of the other oxygen atom to water [14]. CYPs directly participate in the bioconversion of CsA to $\left[\left(4^{\prime}-\mathrm{OH}\right) \mathrm{MeLeu}\right]^{4}$ CsA. In this study, five CYP isoforms (Spot 15, 17-20) were identified and most of them showed higher abundance compared with the control (Table 1). Proteins involved in energy metabolism, especially the redox reaction, displayed a significant improvement (Table 1) under the MO condition. 15 of 16 proteins involved in energy metabolism had higher levels at $48 \mathrm{~h}$, and 10 proteins demonstrated the similar expression pattern at $96 \mathrm{~h}$ (Table 1). In addition, proteins related to the electron transfer chain showed significantly higher levels in the medium MO. In particular, NADH dehydrogenase (Spot 21), the first proton pump in oxidative phosphorylation, showed a 2.4-fold higher abundance than the control at $48 \mathrm{~h}$. Throughout the fermentation process, the abundances of NADH dehydrogenase (Spot 21), FADlinked oxidase (Spot 26) and flavoprotein oxidoreductase (Spot 27) were significantly increased (>3.1-folds) in the medium MO. It is worth noting that NADH dehydrogenase showed the maximum change (20.9-folds) among all the identified proteins. Since the growth of Nonomuraea sp. is strictly dependent on the aerobic metabolism and oxidative phosphorylation [34], as the key members of electron transfer chain, these proteins with higher levels would enhance the accessibility and the turnover rate of $\mathrm{NADH}$ and FADH to CYPs so as to improve the hydroxylation activity [15].

\section{Transcription profiles of the CYPs under the MO condition and the control condition}

Since CYPs directly participates in the monooxygenation reaction, the transcriptional level of CYPs is one of 
the key factors limiting the conversion efficiency. In this study, all the transcriptional expression of CYPs were detected to investigate the impact of soybean oil addition on the CYPs. Previous work has reported that there are 21 species of CYP-sb, among which sb21 plays a leading role in the bioconversion of CsA, and then is CYP-sb22, CYP-sb13, CYP-sb7, and CYP-sb8, etc. according to the single-gene knockout results [14].

The sampling time points $36 \mathrm{~h}$ (12 h after soybean oil addition) and $48 \mathrm{~h}$ ( $24 \mathrm{~h}$ after soybean oil addition) were selected since the greatest specific production rate appeared before $48 \mathrm{~h}$ under both conditions. As shown in Table 2, only 11 CYP genes were detected under both conditions while the other 10 genes were detected only in certain conditions. At $36 \mathrm{~h}$, not only the number of the genes but also the transcription abundance of the CYPs in the soybean oil group were significantly increased compared with the control group (Table 2), which could explain why the specific production rate of the soybean oil group was much higher than that of the control group at $36 \mathrm{~h}$.
At $48 \mathrm{~h}$, the number of the detected CYPs in the control group was larger than that of the soybean oil group, but the transcription levels of the crucial CYP gene sb21, sb13 and sb3-2 were still much lower than the soybean oil group, which could explain the phenomenon that in Fig. 1c the specific production rate of the control group at $48 \mathrm{~h}$ was increased, but was still lower than that in the $\mathrm{MO}$ condition. In addition, the upregulation of the transcription level of the CYPs under the MO condition also implied the formation of the CYP-CsA complex since the TMC (total mole concentration) in the fermentation broth reached bottom at $48 \mathrm{~h}$ and the TMC in the $\mathrm{MO}$ medium was lower than that in the MC medium as shown in Fig. 1d.

Interestingly, the transcription level of CYP-sb21 in the control group greatly reduced at $48 \mathrm{~h}$ (Table 2) but the catalytic efficiency reached the maximum (Fig. 1c). Although sb21 played a leading role in the conversion of CsA to [(4'-OH)MeLeu $]^{4}$-CsA [14], yet the role of other CYPs should not be neglected because the expression of sb3-1, sb4, sb7, sb10, sb16, sb17, sb23 and sb24, and the

Table 2 Transcription profiles of CYPs under the soybean oil and the control conditions

\begin{tabular}{|c|c|c|c|c|c|c|}
\hline \multirow[t]{2}{*}{ Gene name } & \multicolumn{4}{|l|}{$\Delta \mathrm{Ct}_{\mathrm{C} 1}$} & \multicolumn{2}{|l|}{$-\Delta \Delta \mathrm{Ct}$} \\
\hline & $\mathrm{C} 1$ & $\mathrm{C} 2$ & S1 & S2 & S1 vs. $\mathrm{C} 1$ & S2 vs. C2 \\
\hline CYP-sb1 & 19.08 & 16.06 & ND & 18.64 & - & -2.58 \\
\hline CYP-sb10 & ND & 17.28 & ND & ND & - & - \\
\hline CYP-sb11 & 13.01 & 12.45 & 13.00 & 13.36 & 0.01 & -0.91 \\
\hline CYP-sb12 & 6.00 & 9.23 & 3.32 & 8.82 & 2.68 & 0.41 \\
\hline CYP-sb13 & 20.63 & 20.80 & 18.18 & 11.33 & 2.45 & 9.47 \\
\hline CYP-sb15 & 7.05 & 5.38 & 6.39 & 8.24 & 0.66 & -2.86 \\
\hline CYP-sb16 & ND & 20.66 & ND & ND & - & - \\
\hline CYP-sb17 & ND & 17.08 & 14.53 & 10.85 & - & 6.23 \\
\hline CYP-sb2 & 9.16 & 10.90 & 9.79 & 12.55 & -0.63 & -1.65 \\
\hline CYP-sb20 & 9.13 & 10.70 & 9.54 & 8.26 & -0.41 & 2.44 \\
\hline CYP-sb21 & 8.22 & 11.11 & 4.28 & 4.96 & 3.94 & 6.15 \\
\hline CYP-sb22 & 9.78 & 10.04 & 7.20 & 10.83 & 2.58 & -0.79 \\
\hline CYP-sb23 & ND & 12.28 & 13.87 & 12.59 & - & -0.31 \\
\hline CYP-sb24 & ND & $\mathrm{ND}$ & 11.94 & 12.56 & - & - \\
\hline CYP-sb3-1 & ND & 15.17 & ND & ND & - & - \\
\hline CYP-sb3-2 & 16.76 & 11.63 & 12.07 & 8.40 & 4.69 & 3.23 \\
\hline CYP-sb4 & ND & 22.32 & ND & 11.90 & - & 10.42 \\
\hline CYP-sb6 & 19.99 & ND & 14.38 & 17.59 & 5.61 & - \\
\hline CYP-sb7 & ND & 12.85 & 17.41 & ND & - & - \\
\hline CYP-sb8 & 12.15 & 11.64 & 8.32 & 12.31 & 3.83 & -0.67 \\
\hline CYP-sb9 & 9.35 & 8.91 & 7.89 & 10.13 & 1.46 & -1.22 \\
\hline
\end{tabular}

$\mathrm{C} 1, \mathrm{C} 2, \mathrm{~S} 1$ and S2 stand for the samples of 36 and $48 \mathrm{~h}$ under the control condition and the soybean oil condition, respectively $\Delta \mathrm{Ct}$ : The difference value of Cycle Time between the targeted gene and the reference gene. $\Delta \mathrm{Ct}=\mathrm{Ct}($ Targeted gene) $-\mathrm{Ct}(16 \mathrm{~S} r \mathrm{NA})$. $\mathrm{A}$ lower $\Delta \mathrm{Ct}$ represents a higher relative transcription level. $-\Delta \Delta \mathrm{Ct}=\log _{2}$ (fold change)

Each data was calculated the mean value of six samples (three biological repeations and two technical repeations)

ND not detected 
upregulation of sb3-2, sb8, sb9, sb11 and sb15 counteracted the effect of the down-regulated sb21.

\section{Proposed metabolic mechanism of [(4'-OH)MeLeu $]^{4}-\mathrm{CsA}$ overproduction under the MO condition by $N$. dietziae} Soybean oil could improve the production of antibiotics, such as tetracycline, cephamycin $\mathrm{C}$ and tacrolimus [18-20], but the specific mechanism was not always the same. Soybean oil was found to enhance tetracycline production by extracting antibiotic into the oil phase of the culture broth, thereby relieving product inhibition and decreasing damage to microbial cells by foam formation [18]. While in cephamycin C production, soybean oil mainly functioned as a carbon source for Streptomyces sp. p6621 fermentation [19]. Additionally, soybean oil could induce the expression of lipase to produce CoAesters, the precursors of FK506 by $S$. tsukubaensis [20, $22]$. These three products have a similarity that they are produced by the de novo synthesis pathway. However, $\left[\left(4^{\prime}-\mathrm{OH}\right) \mathrm{MeLeu}\right]^{4}-\mathrm{CsA}$ is converted from CsA by the monooxygenation reaction and is directly associated with CYPs, POR (cytochrome P450 reductase), oxygen and reducing equivalent. Thus, the role of soybean oil herein is probably not one of the above mentioned. In this study, the apparent catalytic efficiency (or conversion rate) is closely associated with two factors, i.e., the conversion capacity per unit of the biomass $\left(\mu_{\mathrm{p}}\right)$ and the quantity of biomass $(\mathrm{DCW}) . \mu_{\mathrm{p}}$ is the intrinsic property which is dependent on the abundance of CYPs, PORs and the accessibility of reducing equivalent supplied by the cell metabolism. The synthesis of biomass relies on the abundance of intracellular building blocks, the rate of genetic information processing and the energy availability (ATP and reducing equivalent).

On one hand, the proteomic analysis showed that the significantly changed proteins were involved in EMP, TCA cycle, amino acid metabolism and redox process, thereby enhancing the flux into central carbon metabolism and supplying sufficient cellular building blocks, ATP and reducing equivalents for maintaining the cell robustness and promoting the hydroxylation of CsA. Meanwhile, the improvement of transcription and translation process helps to accelerate the protein synthesis and mycelium growth. Moreover, metabolomic analysis indicated that soybean oil had a great effect on amino acid metabolism and tricarboxylic acid cycle.

On the other hand, the transcriptional analysis of all the CYPs under both conditions confirm that soybean oil can strengthen the CYP system for the conversion of CsA to $\left[\left(4^{\prime}-\mathrm{OH}\right) \mathrm{MeLeu}\right]^{4}-\mathrm{CsA}$. Additionally, the enhanced hydroxylation ability is not only dependent on the elevated expression of CYP-sb21, but also on other CYPs, such as CYP-sb13 and CYP-sb8, although they work with different degrees. These findings demonstrate that the CsA conversion process is under a sophisticated and systematic regulation, although it's a simple in vivo monooxygenation reaction.

Another phenomenon is that besides the soybean oil, some other plant oils, such as corn oil and peanut oil, could also exert a positive influence on the $\left[\left(4^{\prime}-\mathrm{OH}\right)\right.$ MeLeu $]^{4}$-CsA improvement, implying a common feature of these plant oils. In the proteomic analysis, it was noteworthy that no differentially expressed proteins were observed in lipid metabolism (Table 1), implying that the major contribution of soybean oil may not be related to lipid metabolism. As regarding to the metabolomic data, oleic acid, stearic acid, and myristic acid were more abundant in MC samples, especially in the initial fermentation stage (48 and $72 \mathrm{~h}$ ) (Fig. 4). These results demonstrated that lipid metabolism had not been activated under the MO condition. Moreover, the main hydrolysates of soybean oil did not strengthen the CsA conversion ability (Fig. 2). All these results indicated that plant oils could influence the fermentation characteristics. A reasonable explanation was that plant oils increased the oxygen transfer efficiency due to their lower polarity and the stronger oxygen-carrying capacity compared with water [35], thus strengthening the oxygen supply for intracellular redox metabolism and CsA conversion process, just as shown in metabolic pathway analysis in response to soybean oil.

\section{Conclusions}

In summary, a systematic comparative proteomic and metabolomic analysis was successfully conducted to gain insights into the role of soybean oil in improving $\left[\left(4^{\prime}-\mathrm{OH}\right) \mathrm{MeLeu}\right]^{4}-\mathrm{Cs}$ production by $N$. dietziae. Soybean oil could strengthen the primary pathways and the CYP system of $N$. dietziae, thereby improving the rate of biomass synthesis and the hydroxylation efficiency. The omics-based analysis provides a number of intracellular biomarkers, and is a starting point for exploring the regulatory mechanism between cell growth and $\left[\left(4^{\prime}-\mathrm{OH}\right)\right.$ MeLeu $]^{4}$-CsA production, which will be a guidance for the further metabolic engineering of this strain.

\section{Methods}

\section{Microorganism and cultivations}

Nonomuraea dietziae used in this study was stocked in our laboratory and cultivated on ISP-2 agar slant [36]. Seed medium was prepared as Zhang's work [37]. Production medium: $20 \mathrm{~g} / \mathrm{L}$ glucose, $3 \mathrm{~g} / \mathrm{L}$ yeast extract, $10 \mathrm{~g} / \mathrm{L}$ peptone, $12 \mathrm{~g} / \mathrm{L}$ dextrin, $15 \mathrm{~g} / \mathrm{L}$ corn steep liquor and $\mathrm{pH}$ 6.5. $N$. dietziae spores were washed from the fresh agar slant by $5 \mathrm{~mL}$ sterile $0.9 \% \mathrm{NaCl}$ solution and transferred into $100 \mathrm{~mL}$ seed medium of a $500 \mathrm{~mL}$ 
Erlenmeyer flask and then incubated at $28{ }^{\circ} \mathrm{C}, 220 \mathrm{rpm}$ for $72 \mathrm{~h}$. The $\left[\left(4^{\prime}-\mathrm{OH}\right) \mathrm{MeLeu}\right]^{4}$-CsA production was carried out in $50 \mathrm{~mL}$ medium of a $250 \mathrm{~mL}$ flask at $220 \mathrm{rpm}$ for $120 \mathrm{~h}$ at $30{ }^{\circ} \mathrm{C}$ after inoculating $10 \mathrm{~mL}$ seed culture. $0.1 \%(\mathrm{w} / \mathrm{v})$ soybean oil was fed at the beginning of cultivation in the experimental group. Here, CsA was predissolved in $95 \%$ ethanol solution and added into the cultivation medium at $24 \mathrm{~h}$ with an initial concentration of $600 \mathrm{mg} / \mathrm{L}(499.2 \mu \mathrm{mol} / \mathrm{L})$.

\section{Analytical methods}

The biomass yield was determined by dry cell weight $(\mathrm{DCW})$. For the determination of the concentration of $\mathrm{CsA}$ and $\left[\left(4^{\prime}-\mathrm{OH}\right) \mathrm{MeLeu}\right]^{4}-\mathrm{CsA}, 5 \mathrm{~mL}$ culture fluid was immediately mixed with $5 \mathrm{~mL} 95 \%$ ethanol and shaken intermittently for $1 \mathrm{~h}$. After centrifugation, the supernatant was subjected to HPLC (Agilent 1200, USA) equipped with an Eclipse XDB-C18 column (5 $\mu \mathrm{m} ; 150 \mathrm{~mm} \times 4.6 \mathrm{~mm}$; Agilent Technologies) and a UV detector at $210 \mathrm{~nm}$. The mobile phase was acetonitrile- $0.1 \%$ phosphoric acid water solution $(70: 30, \mathrm{v} / \mathrm{v})$ with a flow rate of $1.0 \mathrm{~mL} / \mathrm{min}$, and the column temperature was $60^{\circ} \mathrm{C}$.

The specific production rate $(\mu \mathrm{p})$ is defined as $\mu \mathrm{p}=\mathrm{dp} /$ $\mathrm{dt} / \mathrm{M}$, and the specific consumption rate is defined as $\mu \mathrm{c}=\mathrm{dc} / \mathrm{dt} / \mathrm{M}$, where $\mathrm{p}$ is the $\left[\left(4^{\prime}-\mathrm{OH}\right) \mathrm{MeLeu}\right]^{4}-\mathrm{Cs} \mathrm{A}$ production and $\mathrm{c}$ is the residual CsA in the fermentation broth, $t$ is fermentation time and $M$ is the dry weight of mycelium. $\mathrm{dp} / \mathrm{dt}$ and $\mathrm{dc} / \mathrm{dt}$ was calculated by the slope calculating plug-in "Tangent.opk" in Origin 8.1 (OriginLab, USA).

TMC represents the total molar concentration of the CsA and $\left[\left(4^{\prime}-\mathrm{OH}\right) \mathrm{MeLeu}\right]^{4}-\mathrm{CsA}$ in the fermentation broth, namely, $\mathrm{TMC}=\mathrm{C}(\mathrm{CsA})+\mathrm{C}(\mathrm{CsA}-\mathrm{OH})$.

\section{Protein extraction and proteomics analysis}

Protein extraction for 2-DE was carried out according to the previous work [38], and the experimental details were present in the Additional file 1 (Protein extraction and proteomics analysis). The protein concentration was measured by Bradford method [39]. 2-DE was performed at least in three biological replications for both control and soybean oil conditions. Isoelectric focusing was implemented using a Multiphor II electrophoresis system (Amersham Pharmacia Biotech, Uppsala, Sweden) at $20{ }^{\circ} \mathrm{C}$ for a total of $71,000 \mathrm{~V}$ h under $20^{\circ} \mathrm{C}(\mathrm{S} 1: 0-500 \mathrm{~V}$, $500 \mathrm{~V}$ h; S2: $500 \mathrm{~V}, 2500 \mathrm{~V}$ h; S3: 500-3500 V, 10,000 V h; S4: $3500 \mathrm{~V}, 50,000 \mathrm{~V}$ h; S5: 3500-500 V, $8000 \mathrm{~V}$ h). For each replicate, $0.8 \mathrm{mg}$ protein was loaded onto a $17 \mathrm{~cm}$ immobilized pH gradient (IPG) strip ( $\mathrm{pH} 4-7$; BioRad Laboratories, USA) mixed with $170 \mu \mathrm{L}$ rehydration buffer (8 M urea, $2 \mathrm{M}$ thiourea, 0.5\% (w/v) CHAPS, $1 \%$ $(\mathrm{w} / \mathrm{v})$ DTT, 0.52\% (w/v) Pharmalyte, and 0.002\% (w/v) bromphenol blue). Prior to the second dimensional electrophoresis, IPG strips were equilibrated in two stages: reduction with DTT, then carboxymethylation with iodoacetamide [40]. The proteins in IPG strips were further separated using $12 \%$ sodium dodecyl sulphate-polyacrylamide gels $(26 \times 20 \mathrm{~cm}$; Ettan DALT Twelve system with a programmable power controller) by Bio-Rad Protean II Xi system (Bio-Rad Laboratories, USA).

The protein staining was followed by Bradford method. The staining solution was composed of Coomassie brilliant blue R250 (0.25\%), methanol (100 mL), milli-Q water $(45 \mathrm{~mL})$ and acetate $(45 \mathrm{~mL})$. The destaining solution was composed of ethanol $(50 \mathrm{~mL})$, acetate $(100 \mathrm{~mL})$ and milli-Q water $(850 \mathrm{~mL})$.

The staining gels were scanned at 300 dpi resolution by Umax Powerlook 2100XL Flatbed Scanner (UMAX Technologies Inc., Dallas, TX, USA) [41]. Subsequently, the image was analyzed with the Bio-Rad PDQuest Basic 2-D image processing software (version 8.0.1). By using PDQuest software, the average ratios and $t$ test values for each spot and a value below 0.05 for $t$ test was regarded as being significant. Protein spots with an average abundance change of greater than 1.5-fold and present in all biological replicates were subjected to MS analysis.

Protein spots were detained and digested as previously described [41]. More details were present in Additional file 1 (Protein extraction and proteomics analysis). The digested peptides were analyzed using a 4700 Proteomics Analyzer (Applied Biosystems, USA). The instrument was performed at a maximum accelerating potential of $20 \mathrm{kV}$ and an $m / z$ range from 700 to 4000 . Six standards (Applied Biosystems, USA) were used as the internals to calibrate each spectrum to a mass accuracy within $0.1 \mathrm{Da}$. Protein candidate spots were analyzed using MALDI-TOF/TOF-MS in positive ion mode. Because the database of $N$. dietziae was uncompleted, proteins were identified by automated peptide mass fingerprinting using the Global Proteome Server Explorer software 3.0 (Applied Biosystems, USA) against a self-built protein sequence database of $N$. candida, $N$. coxensis DSM 45129 and Nonomuraea sp. SBT364 and some cyclosporinespecific $\mathrm{P} 450$ hydroxylases. For the algorithm, parameter settings were described by Wang et al. [42]. All the proteins identified were presented by MASCOT report protein scores for MS or total ion scores for MS/MS with greater than $95 \%$ confidence intervals.

\section{Sample preparation of intracellular metabolites for GC-MS}

The experimental data was obtained from four replicates of each treatment. The samples of mycelium at 48,72 , 96 and $120 \mathrm{~h}$ were harvested for quenching and extraction of intracellular metabolites at a low temperature. Subsequently, the extracts were derived with a two-step 
method. The methods of sample preparation for GCMS had been previously described [43] in the Additional file 1 (sample preparation of intracellular metabolites).

\section{Data acquisition and processing of GC-MS}

GC-MS was performed by Agilent 6890N-5975C MSD system (Agilent Technologies, USA) equipped with a DB5MS capillary column $(30 \mathrm{~m} \times 0.25 \mathrm{~mm}, 0.25 \mu \mathrm{m}$ film thickness; Agilent Technologies, USA) and an autosampler. Parameter settings of GC-MS system were consistent with Wang' work [44]. Metabolomic data was processed with the Agilent MSD ChemStation (Agilent Technologies, USA) for spectrum deconvolution, denoising, retention time aligning, peak area integration, compound identification combined with NIST mass spectrum database (http://webbook.nist.gov/chemistry/) [44].

\section{Gene expression determination by quantitative real time RT-PCR (qRT-PCR)}

Real-time PCR was performed to detect the relative transcriptional expression levels of cytochrome $\mathrm{P} 450$ hydroxylase genes of $N$. dietziae. The samples were harvested at 36 and $48 \mathrm{~h}$ from the broth cultivation. Total RNA was extracted with RNA prep pure Cell/Bacteria Kit (TIANGEN, Beijing, China) according to the manufacturer's protocol. The optical density at 260 and $280 \mathrm{~nm}$ was measured to determine the quantity and purity of RNA. cDNA was obtained by reverse transcription with total RNA as template using PrimeScript ${ }^{\mathrm{TM}}$ RT reagent Kit (Takara, Dalian, China) under instructions. On the basis of the CYP gene sequences of $N$. dietziae, the primer pairs were designed and listed in Additional file 1: Table S1, and the gene 16S rDNA was used as the internal control. Then, qRT-PCR analysis was operated in a 7500 Real-Time PCR Systems (Applied Biosystems, USA) with the TransStart Top Green qPCR SuperMix (TransGen Biotech, China) according to the manufacturer's protocol by $2^{-\Delta \Delta \mathrm{Ct}}$ method [45]. Three biological repetitions and two technical repetitions were implemented for each target gene.

\section{Additional file}

Additional file 1: Figure S1. HPLC analysis of CsA and [(4'-OH)MeLeu $]^{4}$ CSA. Figure S2. NMR analysis of CSA and $\left[\left(4^{\prime}-\mathrm{OH}\right) \mathrm{MeLeu}\right]^{4}-\mathrm{CsA}$. Figure S3. HRMS analysis of CSA and [(4'-OH)MeLeu $]^{4}-$ CSA. Figure S4. 2DE-based proteomic profiles of $\mathrm{N}$. dietziae. Proteins are extracted at different growth phases and media. Arrows point to the significantly differential proteins under MO condition and their characteristics are shown in Table 1. Table S1. Primers for qRT-PCR of the CYPs.

\section{Abbreviations}

CSA: cyclosporin A; HIV: human immunodeficiency virus; ISP2 medium: yeast extract-malt extract agar; DCW: dry cell weight; 2-DE: two-dimensional electrophoresis; GC-MS: gas chromatography-mass spectrometry; CYP: cytochrome P450 hydroxylase; EMP: Embden-Meyerh pathway; TCA: tricarboxylic acid; PPP: pentose phosphate pathway; ED pathway: Entner-Doudoroff pathway; G6PD: glucose-6-phosphate dehydrogenase; Edd: phosphogluconate dehydratase; PFK: 6-phosphofructokinase; ENO: enolase; PK: pyruvate kinase; DLD: dihydrolipoamide dehydrogenase; PDH: pyruvate dehydrogenase; CS: citrate synthase; KorA: 2-oxoglutarate ferredoxin oxidoreductase; SucA: alpha-ketoglutarate decarboxylase; sucD: succinyl-CoA synthetase alpha subunit; ASL: argininosuccinate lyase; NDH: NADH dehydrogenase; ALD: alanine dehydrogenase; LeuA: 2-isopropylmalate synthase; GLT: glutamate synthase; UROC1: urocanate hydratase; HAL: histidine ammonia-lyase; KDPG: 2-keto-3-deoxy-6-phosphogluconate; PBS: phosphate buffered solution; PMSF phenylmethanesulfonyl fluoride; DTT: dithiothreitol; IPG: immobilized pH gradient; CHAPS: 3-[(3-cholamidopropyl) dimethylammonio]-1-propanesulfonate.

\section{Authors' contributions}

$\mathrm{HL}$ and $\mathrm{L} J$ conceived and designed the research. $\amalg$ carried out the cell cultivation, proteomic and metabolomic experiment operations, and drafted the manuscript. HL analyzed intracellular metabolites and conducted the RTqPCR array, and contributed to the organization and structure of manuscript. DH contributed to the proteomic data analysis. CW and SL helped to revise the manuscript. JW supervised the research and revised the manuscript. All authors read and approved the final manuscript.

\section{Author details}

${ }^{1}$ Key Laboratory of System Bioengineering (Tianjin University), Ministry of Education, Tianjin 300072, People's Republic of China. ${ }^{2}$ SynBio Research Platform, Collaborative Innovation Center of Chemical Science and Engineering (Tianjin), School of Chemical Engineering and Technology, Tianjin University, Tianjin 300072, People's Republic of China. ${ }^{3}$ TEDA Institute of Biological Sciences and Biotechnology, Nankai University, TEDA, Tianjin 300457, People's Republic of China. ${ }^{4}$ SynBio Research Platform, Collaborative Innovation Center of Chemical Science and Engineering (Tianjin), Nankai University, Tianjin 300071, People's Republic of China.

\section{Acknowledgements}

This work was supported by the Key Program of National Natural Science Foundation of China (No. 21236005), the National Natural Science Foundation of China (No. 21376171 \& 21676189), the National 973 Project of China (No. 2013CB733600), the key technologies R \& D program of Tianjin (No. 16YFZCSY00780).

\section{Competing interests}

The authors declare that they have no competing interests.

\section{Publisher's Note}

Springer Nature remains neutral with regard to jurisdictional claims in published maps and institutional affiliations.

Received: 27 March 2017 Accepted: 10 July 2017

Published online: 14 July 2017

\section{References}

1. Survase SA, Kagliwal LD, Annapure US, Singhal RS. Cyclosporin A-a review on fermentative production, downstream processing and pharmacological applications. Biotechnol Adv. 2011;29(4):418-35.

2. Watashi K, Hijikata M, Hosaka M, Yamaji M, Shimotohno K. Cyclosporin A suppresses replication of hepatitis $C$ virus genome in cultured hepatocytes. Hepatology. 2003;38(5):1282-8.

3. Nakagawa M, Sakamoto N, Enomoto N, Tanabe Y, Kanazawa N, Koyama T, Kurosaki M, Maekawa S, Yamashiro T, Chen CH, et al. Specific inhibition of hepatitis $\mathrm{C}$ virus replication by cyclosporin A. Biochem Biophys Res Commun. 2004;313(1):42-7.

4. Saini M, Potash MJ. Novel activities of cyclophilin A and cyclosporin A during HIV-1 infection of primary lymphocytes and macrophages. J Immunol. 2006;177(1):443-9.

5. Scribner A, Houck D, Huang Z, Mosier S, Peel M, Scorneaux B. Synthesis and biological evaluation of [D-lysine $]^{8} \mathrm{Cyclosporin} A$ analogs as potential anti-HCV agents. Bioorg Med Chem Lett. 2010;20(22):6542-6. 
6. Peel M, Scribner A. Cyclophilin inhibitors as antiviral agents. Bioorg Med Chem Lett. 2013;23(16):4485-92.

7. Kim SN, Ahn H, Lee C, Lee MH, Kim JH, Kim J, Kim S, Cho H, Lee H, Kim H. Use of nonimmunosuppressive [Y'-hydroxy-n-methyl--leucine $\left.{ }^{4}\right]$ cyclosporin derivatives for treating hair loss US Patent 20,040,161,399. 2004.

8. Sweeney ZK, Fu J, Wiedmann B. From chemical tools to clinical medicines: nonimmunosuppressive cyclophilin inhibitors derived from the cyclosporin and sanglifehrin scaffolds. J Med Chem. 2014;57(17):7145-59.

9. Peel M, Scribner A. Semi-synthesis of cyclosporins. Biochim Biophys Acta. 2015;1850(10):2121-44.

10. Woodward DF, Garst ME. Cyclosporin derivatives for enhancing the growth of hair US Patent Application 12,785,158 2010.

11. Kim CD, Lee MH, Sohn KC, Kim JM, Li SJ, Rang MJ, Roh SS, Oh YS, Yoon $\mathrm{TJ}$, Im M, et al. Induction of synapse associated protein 102 expression in cyclosporin A-stimulated hair growth. Exp Dermatol. 2008;17(8):693-9.

12. Takahashi T, Kamimura A. Cyclosporin a promotes hair epithelial cell proliferation and modulates protein kinase $C$ expression and translocation in hair epithelial cells. J Invest Dermatol. 2001;117(3):605-11.

13. Kuhnt M, Bitsch F, France J, Hofmann H, Sanglier JJ, Traber R. Microbial biotransformation products of cyclosporin A. J Antibiot (Tokyo). 1996;49(8):781-7.

14. Lee M-J, Kim H-B, Yoon YJ, Han K, Kim E-S. Identification of a cyclosporinespecific P450 hydroxylase gene through targeted cytochrome P450 complement (CYPome) disruption in Sebekia benihana. Appl Environ Microbiol. 2013;79(7):2253-62.

15. Ma L, Du L, Chen H, Sun Y, Huang S, Zheng X, Kim ES, Li S. Reconstitution of the in vitro activity of the cyclosporine-specific P450 hydroxylase from Sebekia benihana and development of a heterologous whole-cell biotransformation system. Appl Environ Microbiol. 2015;81 (18):6268-75.

16. Lee MJ, Han K, Kim ES. Targeted gene disruption and functional complementation of cytochrome P450 Hydroyxlase involved in cyclosporin A hydroxylation in Sebekia benihana. J Microbiol Biotechnol. 2011;21(1):14-9.

17. Park NS, Myeong JS, Park HJ, Han K, Kim SN, Kim ES. Characterization and culture optimization of regiospecific cyclosporin hydroxylation in rare actinomycetes species. J Microbiol Biotechnol. 2005;15(1):188-91.

18. Jones AM, Porter MA. Vegetable oils in fermentation: beneficial effects of low-level supplementation. J Ind Microbiol Biotechnol. 1998;21(4-5):203-7.

19. Park YS, Momose I, Tsunoda K, Okabe M. Enhancement of cephamycin C production using soybean oil as the sole carbon source. Appl Microbiol Biotechnol. 1994;40(6):773-9.

20. Xia M, Huang D, Li S, Wen J, Jia X, Chen Y. Enhanced FK506 production in Streptomyces tsukubaensis by rational feeding strategies based on comparative metabolic profiling analysis. Biotechnol Bioeng. 2013;110(10):2717-30.

21. Lamb DC, Skaug T, Song HL, Jackson CJ, Podust LM, Waterman MR, Kell DB, Kelly DE, Kelly SL. The cytochrome P450 complement (CYPome) of Streptomyces coelicolor A3(2). J Biol Chem. 2002;277(27):24000-5.

22. Huang D, Xia M, Li S, Wen J, Jia X. Enhancement of FK506 production by engineering secondary pathways of Streptomyces tsukubaensis and exogenous feeding strategies. J Ind Microbiol Biotechnol. 2013;40(9):1023-37.

23. Gunnarsson N, Bruheim P, Nielsen J. Glucose metabolism in the antibiotic producing actinomycete Nonomuraea sp. ATCC 39727. Biotechnol Bioeng. 2004;88(5):652-63.

24. Wilkins MJ, Callister SJ, Miletto M, Williams KH, Nicora CD, Lovley DR, Long PE, Lipton MS. Development of a biomarker for Geobacter activity and strain composition; proteogenomic analysis of the citrate synthase protein during bioremediation of U(VI). Microb Biotechnol. 2011;4(1):55-63.

25. Li L, Wada M, Yokota A. A comparative proteomic approach to understand the adaptations of an $\mathrm{H}^{+}$-ATPase-defective mutant of Corynebacterium glutamicum ATCC14067 to energy deficiencies. Proteomics. 2007;7(18):3348-57

26. Yang Q, Li Y, Yang H, Rang J, Tang S, He L, Li L, Ding X, Xia L. Proteomic insights into metabolic adaptation to deletion of metE in Saccharopolyspora spinosa. Appl Microbiol Biotechnol. 2015;99(20):8629-41.

27. Henard CA, Bourret TJ, Song M, Vazquez-Torres A. Control of redox balance by the stringent response regulatory protein promotes antioxidant defenses of Salmonella. J Biol Chem. 2010;285(47):36785-93.

28. Födinger $M$, Hörl WH, Sunder-Plassmann G. Molecular biology of 5, 10-methylenetetrahydrofolate reductase. J Nephrol. 1999;13(1):20-33.
29. Gunnarsson N, Mortensen UH, Sosio M, Nielsen J. Identification of the Entner-Doudoroff pathway in an antibiotic-producing actinomycete species. Mol Microbiol. 2004;52(3):895-902.

30. Fontecave M, Atta M, Mulliez E. S-adenosylmethionine: nothing goes to waste. Trends Biochem Sci. 2004;29(5):243-9.

31. Caldas T, Laalami S, Richarme G. Chaperone properties of bacterial elongation factor EF-G and initiation factor IF2. J Biol Chem. 2000;275(2):855-60

32. Caldas TD, El Yaagoubi A, Richarme G. Chaperone properties of bacterial elongation factor EF-Tu. J Biol Chem. 1998;273(19):11478-82.

33. Belfiore C, Ordonez OF, Farias ME. Proteomic approach of adaptive response to arsenic stress in Exiguobacterium sp. S17, an extremophile strain isolated from a high-altitude Andean Lake stromatolite. Extremophiles. 2013;17(3):421-31.

34. Palese LL, Gaballo A, Technikova-Dobrova Z, Labonia N, Abbrescia A, Scacco S, Micelli L, Papa S. Characterization of plasma membrane respiratory chain and ATPase in the actinomycete Nonomuraea sp. ATCC 39727. FEMS Microbiol Lett. 2003:228(2):233-9.

35. Clarke KG, Correia LDC. Oxygen transfer in hydrocarbon-aqueous dispersions and its applicability to alkane bioprocesses: a review. Biochem Eng J. 2008;39(3):405-29.

36. Shirling EB, Gottlieb D. Methods for characterization of Streptomyces species. Int J Syst Bacteriol. 1966;16(3):313-40.

37. Zhang L, Dai M, Zheng G, Zhao Y, Liu J, Zhang J, Wang F. Screening and breeding of high producing strain for [Y-HyMeLeu $\left.{ }^{4}\right]$ CyA by ARTP-UV composite mutagenesis. Chin J Microbiol. 2014;39(4):249-52.

38. Martinez-Moya P, Niehaus K, Alcaino J, Baeza M, Cifuentes V. Proteomic and metabolomic analysis of the carotenogenic yeast Xanthophyllomyces dendrorhous using different carbon sources. BMC Genom. 2015;16:289.

39. Bradford MMA. A rapid and sensitive method for the quantitation on microgram quantities of protein utilizing the principle of protein-dye binding. Anal Biochem. 1976;25(1):248-56.

40. Wang C, Chen J, Hu WJ, Liu JY, Zheng HL, Zhao F. Comparative proteomics reveal the impact of OmcA/MtrC deletion on Shewanella oneidensis MR-1 in response to hexavalent chromium exposure. Appl Microbiol Biotechnol. 2014;98(23):9735-47.

41. Zhao G, Hou L, Yao Y, Wang C, Cao X. Comparative proteome analysis of Aspergillus oryzae 3.042 and A. oryzae 100-8 strains: towards the produc tion of different soy sauce flavors. J Proteomics. 2012;75(13):3914-24.

42. Wang J, Liu H, Huang D, Jin L, Wang C, Wen J. Comparative proteomic and metabolomic analysis of Streptomyces tsukubaensis reveals the metabolic mechanism of FK506 overproduction by feeding soybean oil. Appl Microbiol Biotechnol. 2017;101:2447-65.

43. Liu H, Huang D, Wen J. Integrated intracellular metabolic profiling and pathway analysis approaches reveal complex metabolic regulation by Clostridium acetobutylicum. Microb Cell Fact. 2016;15(1):36.

44. Wang B, Liu J, Liu H, Huang D, Wen J. Comparative metabolic profiling reveals the key role of amino acids metabolism in the rapamycin overproduction by Streptomyces hygroscopicus. J Ind Microbiol Biotechnol. 2015;42(6):949-63.

45. Livak KJ. Analyzing real-time PCR data by the comparative $C(T)$ method. Nat Protoc. 2008;3(6):1101-8.

\section{Submit your next manuscript to BioMed Central and we will help you at every step:}

- We accept pre-submission inquiries

- Our selector tool helps you to find the most relevant journal

- We provide round the clock customer support

- Convenient online submission

- Thorough peer review

- Inclusion in PubMed and all major indexing services

- Maximum visibility for your research

Submit your manuscript at www.biomedcentral.com/submit 